\title{
Temporal trends in percutaneous coronary intervention outcomes among older patients in the United States
}

\author{
Sunil V. Rao, MD, Connie N. Hess, MD, David Dai, PhD, MS, Cynthia L. Green, PhD, Eric D. \\ Peterson, MD, MPH, and Pamela S. Douglas, MD \\ Duke Clinical Research Institute, Duke University Medical Center, Durham, NC
}

\section{Abstract}

Background-New percutaneous coronary intervention (PCI) device technologies are often rapidly adopted into clinical practice, yet few studies have examined the overall impact of these new technologies on patient outcomes in community practice.

\begin{abstract}
Methods-In hopes of determining temporal trends in PCI outcomes, we used data from the Centers for Medicare \& Medicaid Service's Chronic Condition Warehouse $(n=3,250,836)$ by comparing patient characteristics and rates of 3-year major adverse cardiac events (MACE) across the balloon angioplasty (POBA) era (01/1991-09/1995), the bare metal stent (BMS) era (02/1998-04/2003), and the drug-eluting stent (DES) era (05/2004-10/2006). The adjusted association between era and outcomes was determined with Cox proportional hazards modeling (POBA era as reference).

Results-Compared with the POBA era, patients undergoing PCI were significantly older and had more medical comorbidities, and the risk for 3-year MACE was significantly lower during the BMS and DES eras (BMS vs. POBA adjusted HR [95\% CI]: 0.930 [0.926-0.935]; DES vs. BMS: 0.831 [0.827-0.835]). Compared with males, the adjusted risk for 3-year MACE among females was lower during the POBA era, but slightly higher during the BMS and DES eras. Across all three eras, patients $\geq 75$ years of age had higher adjusted risk for MACE compared with younger patients, and the risk for revascularization was lower for both females and older patients.
\end{abstract}

Conclusions-Despite its application in older and sicker Medicare beneficiaries, there has been a significant decrease in post-PCI MACE over time. The risk for death or myocardial infarction is higher among females and older patients compared with males and younger patients; therefore, future studies should focus on improving clinical outcomes in these high-risk subgroups.

\footnotetext{
(C) 2013, Mosby, Inc. All rights reserved.

Reprint requests: Sunil V. Rao, MD; 508 Fulton Street (111A), Durham, NC 27705. sunil.rao@ duke.edu. Hitinder S. Gurm, MBBS, served as guest editor for this article.

Conflict of Interest Disclosures: SV Rao: Dr Rao reports research funding: Cordis Corporation; Consultant/Honoraria: The Medicines Company, Zoll, Terumo Medical, Astra Zeneca, Daiichi Sankyo Lilly, Bristol Myers Squibb, Sanofi-Aventis.

CN Hess: Dr Hess has no disclosures to report.

D Dai: Dr Dai has no disclosures to report.

CL Green: Dr Green has no disclosures to report.

ED Peterson: Please see www.dcri.org for a complete list of Dr Peterson's disclosures.

PS Douglas: Dr Douglas has no disclosures to report.
} 
Percutaneous coronary intervention (PCI) is one of the most commonly performed cardiac procedures among Medicare recipients. Since its invention, there has been considerable evolution in device technology that, in clinical trials, has led to decreased risks for procedural complications and the need for repeat revascularization. ${ }^{1-3}$ Many new devices, or even iterations of existing devices, are rapidly taken up into clinical practice; this was especially true for drug-eluting stents (DES), where the uptake was particularly brisk. ${ }^{4}$ Yet to date, few studies have examined the overall impact of these new PCI technologies on patient outcomes in community practice. Available data on temporal trends in PCI outcomes in the United States are from either single center, ${ }^{5}$ regional, ${ }^{6}$ or small multicenter registries, ${ }^{7}$ and demonstrate improved outcomes over time. While these data are reassuring, trends could reflect other practice patterns specific to the centers studied and may not be broadly representative. Moreover, outcome patterns in higher risk groups such as females and the elderly remain unclear.

Accordingly, we examined a large administrative database to examine temporal trends in PCI outcomes. We created three distinct PCI "eras" defined by: (1) balloon angioplasty (POBA); (2) bare metal stents (BMS); or (3) DES, and hypothesized that despite the use of PCI in higher risk patients over time, the risks of short- and long-term death or repeat revascularization have decreased. In addition, we compared outcomes over time in 2 key subgroups: females and patients $\geq 75$ years old.

\section{Methods}

\section{Study sample}

The Centers for Medicare \& Medicaid Services launched a research database called the Chronic Condition Data Warehouse (CCW), in response to the Medicare Modernization Act of 2003. The CCW contains fee-for-service institutional and non-institutional claims, assessment data, and enrollment/eligibility information linked by a unique, unidentifiable beneficiary key, which allows researchers to analyze information across the continuum of care. Details on the data are available at: http://www.ccwdata.org.

This study included all Medicare-eligible patients $\ 65$ years of age undergoing PCI in the CCW data from 1991-2008. Only patients without prior coronary artery bypass grafting (CABG) were included $(n=3,250,836)$. We divided the study period into eras defined by use of specific PCI technology: (1) POBA, (2) BMS, and (3) DES. Each era was defined according to the proportion of patients who received POBA, BMS, or DES. Months during which the use of POBA, BMS, or DES was over 75\% defined an era as "POBA," "BMS," or "DES," respectively. The POBA era (POBA use $775 \%$ ) covers between January 1991 and September 1995; the BMS era (BMS use $75 \%$ ) covers between February 1998 and April 2003; and the DES era (DES use $775 \%$ ) covers between May 2004 and October 2006. Demographics and comorbid conditions, including the Deyo-Charlson index ${ }^{8}$ were derived from claims. 


\section{Follow-up information}

The same data were used for longitudinal patient follow-up. Percutaneous coronary intervention procedure codes (Interna-[ICD-9-CM] procedure codes: 00.66, 36.01, 36.02, $26.05,36.05,36.06$, and 36.07 ) were used to identify index procedures and to create longitudinal profiles.

\section{Clinical end points}

We evaluated 3 primary clinical endpoints: death, myocardial infarction (MI), and repeat revascularization, as well as their composites. We also evaluated the mode of repeat revascularization (PCI or $\mathrm{CABG}$ ) as secondary outcomes. Death was defined according to beneficiary death date in the $\mathrm{CCW}$ data, and other clinical end points were defined with the $\mathrm{CCW}$ claims files as the primary diagnosis for a hospital admission. The ICD-9-CM diagnosis codes 410 .X were used to define MI, and ICD-9-CM procedure codes 00.66, $36.00,36.06,36.07,36.09$, and 36.10-36.19 were used to define revascularization.

\section{Statistical analysis}

Baseline characteristics for the study population were provided overall and by PCI era using descriptive statistics (number of observations, mean, standard deviation, median, 25th and 75th percentiles, minimum, and maximum) for numerical (or continuous) variables and with frequency and percentages for categorical variables. Bivariate tests of association were based on either Pearson $\chi^{2}$ tests for categorical variables or Kruskal-Wallis tests for continuous or ordinal variables.

Time to first clinical outcome (eg, death, MI, or repeat revascularization) occurring after the index revascularization episode was computed and plotted based on Gray's method by using cumulative incidence functions that accounted for administrative censoring and included death as a competing risk. ${ }^{9}$ Unadjusted estimates of the clinical event rates at 1 year, 2 years, and 3 years post-intervention were tabulated.

Cox proportional hazards models accounting for competing risks were used to estimate hazard ratios (HRs) and adjusted clinical event rates associated with risk of death first, MI first, and revascularization first with adjustment for baseline variables that were selected a priori based on clinical expertise. ${ }^{10}$ Cox models were also employed to estimate both unadjusted and adjusted HRs for the primary and secondary clinical outcomes separately for the BMS and DES eras using the POBA era as the reference standard. Covariates entered into the models included those listed above. The analyses were repeated to consider sex and patients $\geq 75$ years. In the sex- and age-specific analyses, adjusted HRs for each outcome were generated for the POBA, BMS, and DES eras using males and patients $<75$ years as the reference groups, respectively. We also examined the interaction between era (POBA, BMS, DES) and sex, as well as the interaction between era and age, for each outcome.

All statistical analyses were conducted by the Duke Clinical Research Institute using SAS version 9.2 or higher (SAS Institute, Inc, Cary, NC). All statistical tests were 2 sided with a significance level of .05 , with no adjustment for multiple comparisons. 


\section{Results}

\section{Study sample}

We identified 3,250,836 Medicare recipients who underwent PCI during the study period. The POBA era existed from January 1991 to September 1995; the BMS era, from February 1998 to April 2003; and the DES era, from May 2004 to October 2006 (Figure 1; annualized rates of PCI during the study period are shown in the online Appendix Supplementary Figure 1). Accordingly, 564,565 patients underwent PCI during the POBA era, 1,024,243 patients underwent PCI during the BMS era, and 604,453 patients underwent PCI during the DES era. Table I shows the baseline demographic and medical characteristics of the patients overall, as well as during each era. Over time, the risk profile of patients undergoing PCI increased, with older patients and patients with more medical comorbidities in each era.

\section{Outcomes}

Figure 2A-C displays the unadjusted cumulative incidence rates for clinical outcomes across eras at 1 year, 2 years, and 3 years. The rates of major adverse cardiac events (MACE; death, MI, or revascularization) were lower during the DES era compared with either the BMS or POBA eras. This finding was primarily due to lower rates of repeat revascularization during the BMS and DES eras (Figure 3A-C), and in particular, the rate of subsequent $\mathrm{CABG}$ surgery.

Table II shows the adjusted HRs for the outcomes comparing the BMS era with the POBA era, and the DES era with the BMS era. There was an association between each generation of interventional devices and a lower risk for short- and long-term outcomes (3-year MACE: BMS vs. POBA adjusted HR [95\% CI]: 0.930 [0.926-0.935]; DES vs. BMS: 0.831 [0.827-0.835]). Again, the greatest reduction was in the risk for revascularization by CABG.

\section{Sex and age subgroups}

We also examined outcomes in females vs. males as well as patients aged $<75$ years and $\geq 75$ years within each era. Among females, the cumulative unadjusted rate of MACE at 1,2, and 3 years was lower during the DES era compared with either the BMS or POBA eras; this was driven primarily by a lower risk for subsequent revascularization (online Appendix Supplementary Figures $1 \mathrm{~A}-\mathrm{C}$ and $2 \mathrm{~A}-\mathrm{C}$ ). The unadjusted rates of death were slightly higher at each follow-up timepoint. After adjustment, compared with males, female sex was associated with a lower adjusted risk for MACE during the POBA era (online Appendix Supplementary Table I). However, during the BMS and DES eras, female sex was associated with a higher adjusted risk for MACE. This finding appeared to be driven by an increased adjusted risk for MI during the BMS and DES eras. The adjusted risk for death, repeat PCI, and CABG were lower for females compared with males during all three eras.

The association between age $\geq 75$ years and MACE was higher across all three eras, but again, was not consistent across all outcomes. The adjusted risk for death and MI was higher, but the adjusted risk for revascularization was lower during each era (online Appendix Supplementary Table II; online Appendix Supplementary Figures 3A-C and 4AC). We also examined the interaction between era and sex, as well as era and age, for each 
of the outcomes. The interactions were all significant $(P<.001)$, except for the interaction between era and sex for the outcome of repeat PCI $(P=.46)$.

\section{Discussion}

Our examination of this large cohort of Medicare beneficiaries resulted in three key findings: (1) over time, PCI has been performed in patients with more medical comorbidities; (2) despite this higher risk profile, both short- and long-term outcomes improved over time; and (3) compared with males and patients younger than 75 years, female sex and those patients (males or females) $\geq 75$ years have a higher risk for MACEprimarily driven by either MI or death and MI. The risk for revascularization was lower for both subgroups across all three eras. These data suggest that advancements in PCI technology, including both devices and pharmacology, have had a positive impact on important post-procedure clinical events, and future efforts should focus on improving clinical outcomes such as MI in older patients and females.

Prior studies have come to similar conclusions, but have examined limited datasets. Singh et al. evaluated outcomes after PCI over a 25-year period at the Mayo Clinic. ${ }^{5}$ Similar to our study, the investigators found that over time, PCI was performed in patients with a greater number of comorbid conditions including prior percutaneous coronary procedures. Procedural success improved significantly over time, and the rates of emergency CABG and long-term MACE decreased significantly. These outcomes likely reflect a combination of interventional expertise and care processes at this single center, as well as the evolution in PCI drugs, devices, and secondary prevention. One multicenter study on temporal trends in outcomes is from the National Heart Lung and Blood Institute's (NHLBI) Balloon Angioplasty Registry and Dynamic Registry. ${ }^{7}$ Data on 8976 patients from the Balloon Angioplasty Registry and 4 waves of the Dynamic Registry's enrollment (representing BMS, routine use of stents, brachytherapy, and DES) demonstrated significant reductions in inhospital MI and emergency CABG, as well as reductions in the risks of 1-year revascularization. Trikalinos et al. conducted a network meta-analysis of 61 clinical trials of non-acute PCI comparing POBA, BMS, DES, and medical therapy. ${ }^{11}$ The investigators found no reductions in death or MI but found significant reductions in both target vessel and target lesion revascularization as technology evolved from POBA to BMS to DES. Our study extends these prior studies in 2 ways: (1) by examining a large broadly representative group of patients encountered in clinical practice across multiple centers, including those undergoing PCI for non-acute indications; and (2) by examining 3-year outcomes including death, MI, and revascularization by either PCI or CABG.

We found reductions in death and MI between the POBA and BMS eras and reductions in death, MI, and revascularization between the BMS and DES eras. These trends likely reflect a variety of practice changes over time, including the widespread use of proven primary and secondary prevention measures such as statins and smoking cessation. Indeed, these noninterventional therapies may have had a greater impact on improved outcomes than device technology, per se. From an interventional perspective, our finding of an increased risk for revascularization between the POBA and BMS eras may represent the use of stenting for "bailout" during the early BMS era before the widespread use of direct routine stenting. 
With respect to reductions in death and MI, we separated time periods defined by interventional devices (POBA, BMS, and DES), but there was a concomitant evolution in procedural pharmacology and secondary prevention strategies after PCI. For example, antithrombotic strategies have changed significantly over time to include newer anticoagulants and antiplatelet agents. ${ }^{12}$ In addition, there has been greater focus on periprocedural bleeding complications, which may also impact outcomes. ${ }^{13,14}$ Therefore, the finding of an association between each iteration in PCI technology and death should be interpreted with caution since we did not compare PCI with medical therapy, and the aforementioned evolution in secondary prevention could account for the decrease in mortality.

While the changes in PCI practice appear to have resulted in better outcomes for most patients, we found interesting trends among females and patients $\geq 75$ years of age. Females had lower risks for all outcomes except for MI, although this risk was only modestly increased. Singh et al have previously shown that 30-day and 1-year mortality was similar between men and women undergoing PCI at the Mayo Clinic. ${ }^{15}$ The investigators were unable to show any differences in 1-year MI or repeat revascularization in their single center study. A large study examining sex-based differences in outcomes by stent type among Medicare beneficiaries has shown that while women have higher pre-procedural risk compared with men, they have higher long-term survival. ${ }^{16}$ The use of DES was associated with significant reductions in long-term death, MI, or revascularization. In the context of these data, our findings may reflect disparities in the use of proven secondary prevention strategies between males and females. We also examined outcomes among patients $\geq 75$ years of age compared with younger patients and found that while the risks of MACE were higher during all three eras, this risk was driven by an increased risk for death; whereas the risks for $\mathrm{MI}$ and revascularization were lower. When compiled together, these data suggest that the advances in device technology and medical therapy resulted in lower rates of repeat procedures, especially in the rate of CABG. The reduction in the rate of CABG after PCI was of larger magnitude than other outcomes and is likely specifically reflective of stent efficacy. On the other hand, the differences in other clinical outcomes may reflect differential application of evidence-based secondary prevention strategies among females and the elderly, ${ }^{17,18}$ adherence to such strategies, bleeding risk, or a combination of these processes. Importantly, there was an increase in the number of PCI procedures from the POBA era to the BMS era to the DES era; this, coupled with the increased number of comorbidities from era to era, indicates that threshold for selecting patients for PCI likely went lower as technology evolved. Therefore, some of the poor outcomes may reflect the higher baseline risk of the females and older patients who underwent PCI during the BMS and DES eras. Nevertheless, future studies should focus on elucidating the mechanisms underlying the outcomes in these two subgroups and consequent interventions to reduce their risk. Such efforts may include the study of radial access or vascular closure devices to reduce periprocedural bleeding, ${ }^{19}$ as well as clinical trials of DES platforms performed specifically in these populations. There are studies currently in progress (eg, www.clinicaltrials.gov identifiers NCT00496938 and NCT01406236) addressing some of these issues, which have the potential to further improve outcomes after PCI. 


\section{Limitations of the study}

Our study had several limitations. First, we used claims data to examine outcomes. While this allowed for a large broadly representative population to be explored, the data did not contain granular clinical information that could have influenced our models. As a result, it is possible that the increase in patient comorbidities could be due to greater coding of concomitant conditions, changes in the definitions of comorbidities, or both. Second, we did not have information on background secondary prevention therapies or adherence to evidence-based medications - both of which can clearly influence outcomes. Third, while we were able to demonstrate reductions in repeat revascularization (by both repeat PCI or $\mathrm{CABG}$ ), we did not have access to angiographic information; therefore, we cannot definitively comment on whether these revascularization patterns are due to reductions in target vessel or target lesion revascularization. Fourth, while we intended to include patients without prior CABG, we could not reliably do this for the patients identified in 1991. However, since angioplasty was described only a few years prior to 1991, and was initially limited to native vessels, it is unlikely that many CABG patients were represented in our study sample from 1991. Fifth, we relied on claims data to determine comorbidities and temporal changes in coding, as well as variability in coding practices across operators and/or sites, could account for some of the results. Also, since we used claims to assess outcomes, we could not reliably distinguish cardiac from noncardiac mortality. Finally, our study was observational, and despite robust statistical adjustment, unmeasured confounders may have been present.

In conclusion, this large study of Medicare beneficiaries demonstrates that, over time, PCI has increasingly been used in patients with greater medical comorbidities. Furthermore, both short- and long-term major adverse cardiac events have significantly decreased over time. In particular, the risk for revascularization has been significantly reduced; however, compared with males and younger patients, post-PCI clinical outcomes in women and patients $\geq 75$ years of age appear to be worse in the BMS and DES eras-primarily driven by clinical outcomes such as death or MI. Further study is needed to identify strategies to improve clinical outcomes in these two high-risk subgroups.

\section{Supplementary Material}

Refer to Web version on PubMed Central for supplementary material.

\section{Acknowledgments}

The authors would like to thank Erin LoFrese for her editorial assistance with this manuscript. Ms. LoFrese did not receive compensation for her assistance, apart from her employment at the institution where this study was conducted.

Funding Sources: This project was sponsored by the Agency for Healthcare Research and Quality, United States Department of Health and Human Services, Rockville, MD, as part of the Cardiovascular Consortium and funded under Project ID: 24-EHC-1 and Work Assignment Number: HHSAA290-2005-0032-TO4-WA3 as part of the Developing Evidence to Inform Decisions about Effectiveness (DEcIDE) program. The authors of this report are responsible for its content. Statements in the report should not be construed as endorsement by the Agency for Healthcare Research and Quality, nor the United States Department of Health and Human Services.

Open access policy: This research was conducted through a Government contract with the Agency of Healthcare Research and Quality, an operating division of the US Department of Health and Human Services. Approval to 
assert copyright by the authors on the manuscript has been granted by the Government through the attached letter signed by the official Agency of Healthcare Research and Quality Contracting Officer. As stated in the letter, the Government retains rights to the use of the manuscript according to the contract and the Federal Acquisition Regulations. To meet the need for public accessibility to works funded by the Government, the authors request that the journal provide "open access" to the article if it is accepted for publication. Open access means that the final journal article is available electronically for free at the time of publication through the journal's Web site.

Role of the sponsor: The funding organization had no role in the design and conduct of the study; in the collection, analysis, and interpretation of the data; or in the preparation, review, or approval of the manuscript.

\section{References}

1. Stone GW, Ellis SG, Cox DA, et al. A polymer-based, paclitaxel-eluting stent in patients with coronary artery disease. N Engl J Med. 2004; 350:221-31. [PubMed: 14724301]

2. Stone GW, Rizvi A, Newman W, et al. Everolimus-eluting versus paclitaxel-eluting stents in coronary artery disease. N Engl J Med. 2010; 362:1663-74. [PubMed: 20445180]

3. Moses JW, Leon MB, Popma JJ, et al. Sirolimus-eluting stents versus standard stents in patients with stenosis in a native coronary artery. N Engl J Med. 2003; 349:1315-23. [PubMed: 14523139]

4. Rao SV, Shaw RE, Brindis RG, et al. Patterns and outcomes of drug-eluting coronary stent use in clinical practice. Am Heart J. 2006; 152:321-6. [PubMed: 16875917]

5. Singh M, Rihal CS, Gersh BJ, et al. Twenty-five-year trends in in-hospital and long-term outcome after percutaneous coronary intervention: a single-institution experience. Circulation. 2007; 115:2835-41. [PubMed: 17533185]

6. Ko DT, Tu JV, Samadashvili Z, et al. Temporal trends in the use of percutaneous coronary intervention and coronary artery bypass surgery in New York State and Ontario. Circulation. 2010; 121:2635-44. [PubMed: 20529997]

7. Venkitachalam L, Kip KE, Selzer F, et al. Twenty-year evolution of percutaneous coronary intervention and its impact on clinical outcomes: a report from the National Heart, Lung, and Blood Institute-sponsored, multicenter 1985-1986 PTCA and 1997-2006 Dynamic Registries. Circ Cardiovasc Interv. 2009; 2:6-13. [PubMed: 20031687]

8. Deyo RA, Cherkin DC, Ciol MA. Adapting a clinical comorbidity index for use with ICD-9-CM administrative databases. J Clin Epidemiol. 1992; 45:613-9. [PubMed: 1607900]

9. Gray RJ. A class of K-sample tests for comparing the cumulative incidence of a competing risk. Ann Statist. 1988; 16:1141-54.

10. Rosthoj S, Andersen PK, Abildstrom SZ. SAS macros for estimation of the cumulative incidence functions based on a Cox regression model for competing risks survival data. Comput Methods Programs Biomed. 2004; 74:69-75. [PubMed: 14992828]

11. Trikalinos TA, Alsheikh-Ali AA, Tatsioni A, et al. Percutaneous coronary interventions for nonacute coronary artery disease: a quantitative 20-year synopsis and a network meta-analysis. Lancet. 2009; 37:911-8. [PubMed: 19286090]

12. King SB 3rd, Smith SC Jr, Hirshfeld JW Jr, et al. 2007 Focused Update of the ACC/AHA/SCAI 2005 Guideline Update for Percutaneous Coronary Intervention: a report of the American College of Cardiology/American Heart Association Task Force on Practice Guidelines: 2007 Writing Group to Review New Evidence and Update the ACC/AHA/SCAI 2005 Guideline Update for Percutaneous Coronary Intervention, Writing on Behalf of the 2005 Writing Committee. Circulation. 2008; 117:261-95. [PubMed: 18079354]

13. Doyle BJ, Rihal CS, Gastineau DA, et al. Bleeding, blood transfusion, and increased mortality after percutaneous coronary intervention: implications for contemporary practice. J Am Coll Cardiol. 2009; 53:2019-27. [PubMed: 19477350]

14. Rao SV, O'Grady K, Pieper KS, et al. Impact of bleeding severity on clinical outcomes among patients with acute coronary syndromes. Am J Cardiol. 2005; 96:1200-6. [PubMed: 16253582]

15. Singh M, Rihal CS, Gersh BJ, et al. Mortality differences between men and women after percutaneous coronary interventions. A 25-year, single-center experience. J Am Coll Cardiol. 2008; 51:2313-20. [PubMed: 18549915] 
16. Anderson ML, Peterson ED, Brennan JM, et al. Short- and long-term outcomes of coronary stenting in women versus men: results from the National Cardiovascular Data Registry Centers for Medicare \& Medicaid Services cohort. Circulation. 2012; 126:2190-9. [PubMed: 22988009]

17. Bugiardini R, Yan AT, Yan RT, et al. Factors influencing underutilization of evidence-based therapies in women. Eur Heart J. 2011; 32:1337-44. [PubMed: 21383003]

18. Jneid H, Fonarow GC, Cannon CP, et al. Sex differences in medical care and early death after acute myocardial infarction. Circulation. 2008; 118:2803-10. [PubMed: 19064680]

19. Rao SV, Ou FS, Wang TY, et al. Trends in the prevalence and outcomes of radial and femoral approaches to percutaneous coronary intervention: a report from the National Cardiovascular Data Registry. J Am Coll Cardiol Interv. 2008; 1:379-86. 


\section{Overall (Cut Point $=\mathbf{7 5} \%$ )}

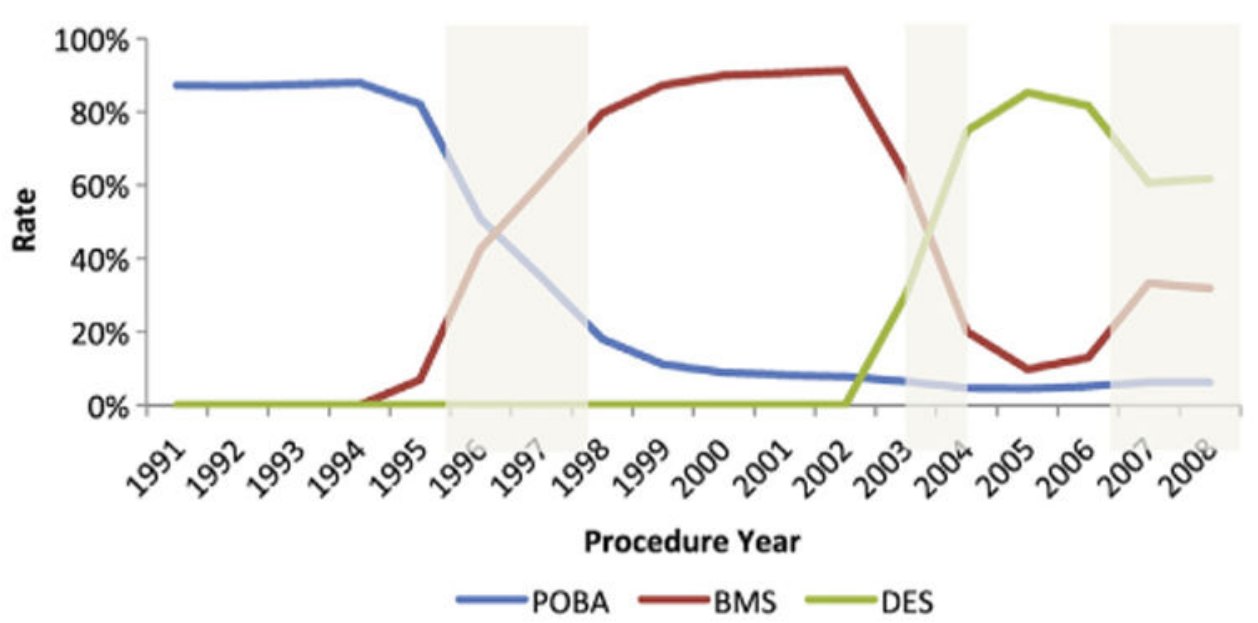

Figure 1.

Delineation of the POBA, BMS, and DES eras. Delineation of eras defined as $\geq 75 \%$ use of POBA, BMS, or DES, respectively. 

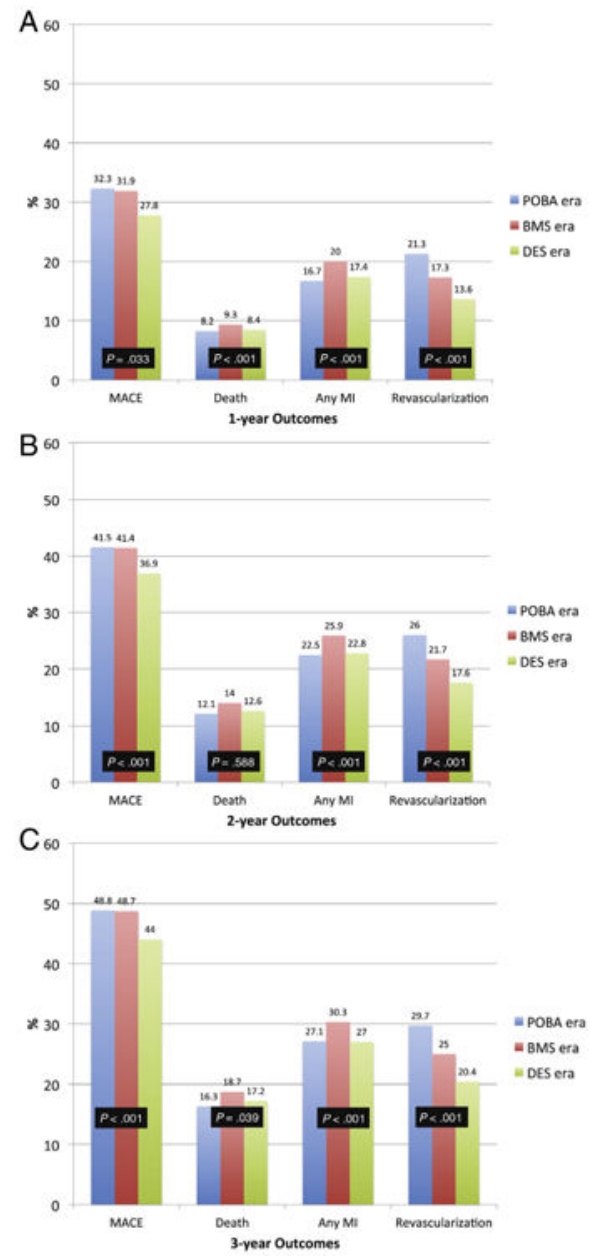

Figure 2.

Cumulative incidence rates of MACE, death, any MI, and revascularization. Cumulative incidence rates of MACE, death, any MI, and revascularization across the POBA, BMS, and DES eras at: A, One-year post index PCI. B, Two years post index PCI. C, Three years post index PCI. Global P values are presented. 

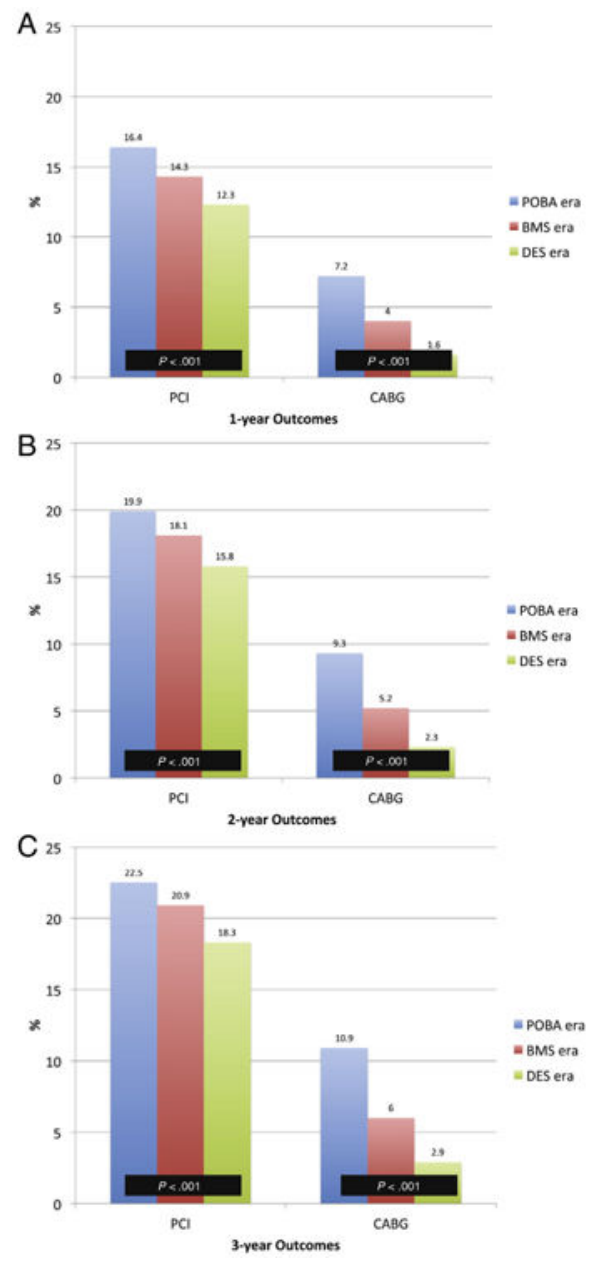

Figure 3.

Cumulative incidence rates of repeat PCI or CABG. Cumulative incidence rates of repeat PCI or CABG across the POBA, BMS, and DES eras at: A, One year post index PCI. B, Two years post index PCI. C, Three years post index PCI. Global P values are presented. 


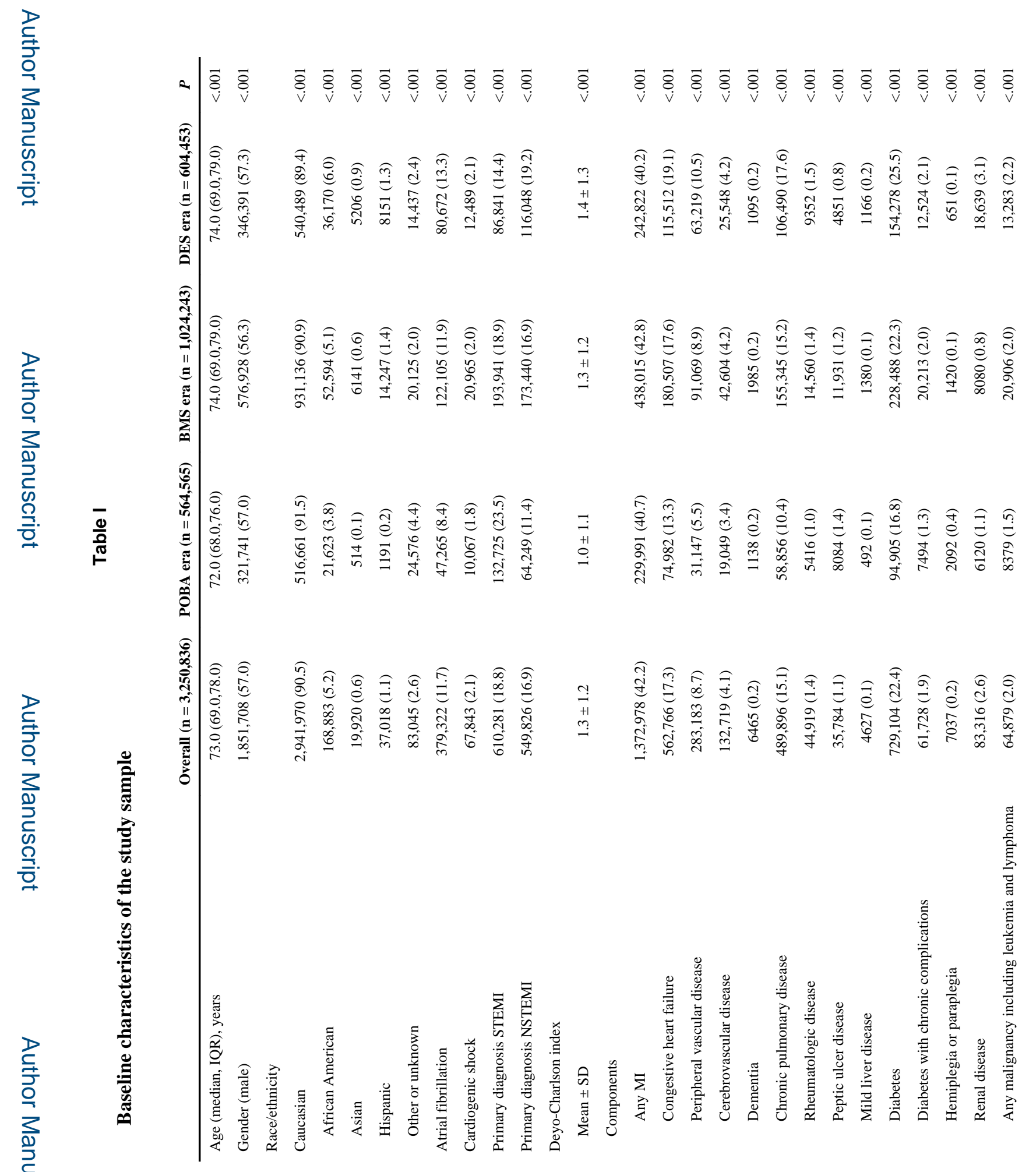

Am Heart J. Author manuscript; available in PMC 2015 April 22. 


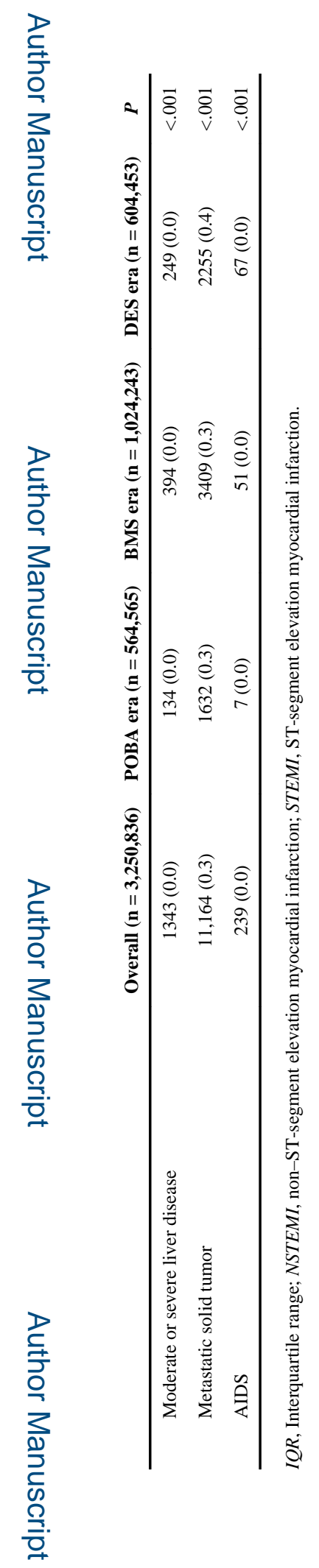

Am Heart J. Author manuscript; available in PMC 2015 April 22. 


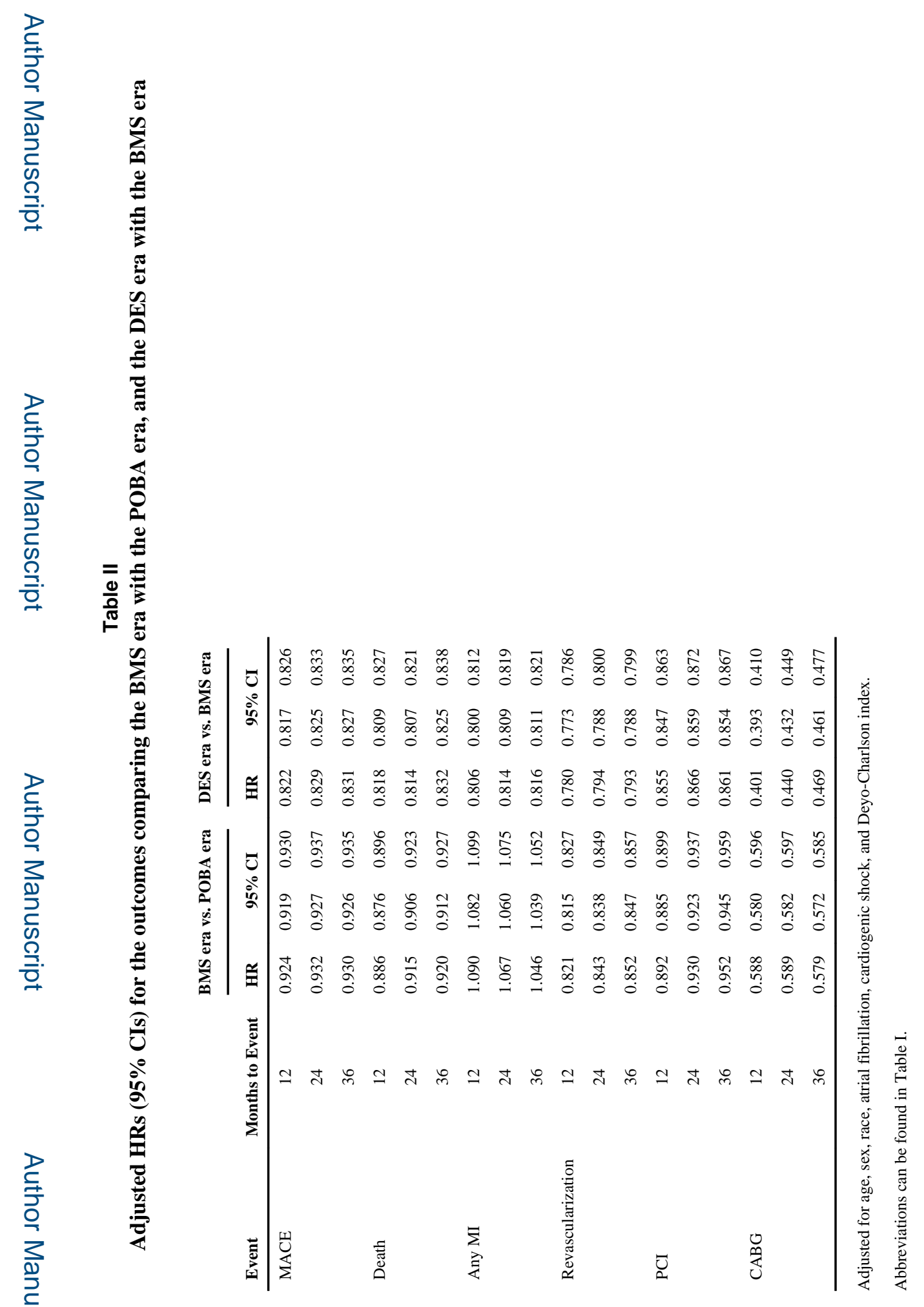

Am Heart J. Author manuscript; available in PMC 2015 April 22. 